\title{
Sobre o Debate Freqüentista Versus Probabilista: "Sorte de Tolo" Torna-se uma Explicação Plausível
}

\author{
Antonio Roazzi $i^{12}$ \\ Universidade Federal de Pernambuco \\ David P. O'Brien \\ Baruch College and the Graduate Center of the City University of New York \\ Maria da Graça B. B. Dias \\ Universidade Federal de Pernambuco
}

\begin{abstract}
Resumo
Um debate entre Kahneman e colaboradores por um lado, e Gigerenzer e colaboradores e Cosmides e colaboradores por outro, tem ocorrido na área de raciocínio sobre probabilidades condicionais. Kahneman e Tversky propuseram que as pessoas tipicamente representam informações em termos de exemplos individuais, e, então, elas fazem julgamentos usando processos de raciocínio que se baseiam em tais exemplos. Cosmides e colaboradores, entretanto, propuseram que as pessoas tipicamente representam informações sobre freqüências populacionais. Uma série de problemas metodológicos nas comparações entre problemas freqüentistas e probabilistas levantadas por Gingerenzer e Hoffrage e por Cosmides e Tooby é discutida. Finalmente, discutimos uma possível estratégia, denominada por O’Brien, Roazzi e Dias de teoria Sorte de Tolo. Este artigo assegura que os problemas de formato freqüentista permitem a existência de uma estratégia de adivinhação que não existe nos problemas de formato probabilista, e levanta a possibilidade de que toda a literatura nesta área tem falsamente assumido que as respostas corretas se originam de apropriadas linhas de raciocínio, enquanto que respostas incorretas não, o que indica, de certa forma, que nem respostas corretas nem incorretas têm se originado de linhas de raciocínio Bayesiano.

Palavras-chave: Raciocínio condicional; raciocínio Bayesiano; formato freqüentista; formato probabilista.
\end{abstract}

On the Frequentist and Probabilistic Debate: "Dumb-Luck" Turns out to be a Plausible Explanation

\begin{abstract}
A debate between Kahneman and Tversky and their associates, on the one hand, and Gigerenzer and his associates and Cosmides and her associates, on the other hand, has been fought in the area of reasoning about conditional probabilities. Kahneman and Tversky proposed that people typically represent information in terms of individual exemplars, and thus people make judgments using reasoning processes that are based on such individual exemplars. Cosmides and Gigerenzer and their associates, however, proposed that people typically represent information about population frequencies. A series of confounds in the comparisons between frequentist and probabilist problems by Gigerenzer and Hoffrage, and by Cosmides and Tooby are discussed.. Finally, we discuss a possible strategy, labeled by O’Brien, Roazzi and Dias as "the dumb-luck theory". This proposal holds that frequentist-formatted problems made available a successful guessing strategy that was not available on the probabilistformatted problems, and the proposal opens the possibility that the entire research literature in this area has falsely assumed that correct answers stem from appropriate lines of reasoning, whereas incorrect answers do not, indicating in such a way that neither the correct nor the incorrect answers have stemmed from Bayesian lines of reasoning at all.

Keywords: Conditional reasoning; Bayesian reasoning; frequentist formatting; probabilistic formatting.
\end{abstract}

\footnotetext{
${ }^{1}$ Os autores agradecem ao CNPq e ao NSF pelos financiamento da pesquisa que tem produzido este artigo: Convênio de Cooperação Bilateral CNPq (Proc. número 910023/01-8) / NSF (National Science Foundation, USA), iniciado a partir de $1^{\circ} \mathrm{de}$ julho de 2001, envolvendo auxílios financeiros tanto nos EUA como no Brasil para realização do Projeto de Pesquisa Conjunta "Perspectiva transculturais e evolutivas na linguagem do pensamento”. O projeto é coordenado no Brasil pelos Professores Antonio Roazzi e Maria da Graça Dias e nos EUA pelos Professores David P. O’Brien e Patricia Brooks. No Brasil o Convênio é gerenciado pela Assessoria de Cooperação Internacional do Conselho Nacional de Desenvolvimento Científico e Tecnológico (ASCIN/CNPq). O número do processo no NSF é INT0104503, U.S.-Brazil Cooperative Research: "Cross Cultural and Cross Linguistic Investigations of Deduction and Quantification".

${ }^{2}$ Endereço para correspondência: Universidade Federal de Pernambuco, CFCH, 8 o andar, Cidade Universitária, Rua Acadêmico Hélio Ramos, s/n, 50670 901, Recife, PE. Fone: (81) 32718272 / 32710599, Fax: (81) 32711843. E-mails: roazzi@ufpe.br, david_obrien@baruch.cuny.edu,mdias@ufpe.br
}

Durante as duas últimas décadas, indagações sobre se os processos de raciocínio humano correspondem a procedimentos normativos têm se tornado um assunto bastante debatido em periódicos de renome como Psychological Review, Behavioral and Brain Sciences, Cognition, Journal of Memory and Language, e Journal of Experimental Psychology: General. Este debate teórico vem acontecendo principalmente em duas áreas de pesquisa: raciocínio dedutivo e raciocínio sobre probabilidades condicionais. Este artigo propõe questionar aspectos levantados por este debate teórico e apontar novas linhas de investigação.

Neste contexto, de um lado tem-se Kahneman e Tversky e seus associados (Ex.: Kahneman, Slovic, \& Tversky, 1982; Kahneman \& Tversky, 1972, 1973, 1996) e, do outro, 
Gigerenzer e seus associados (Ex.: Gigerenzer, 1996; Gigerenzer \& Hoffrage, 1995), junto com Cosmides e seus associados (Ex.: Cosmides \& Tooby, 1996). Tal debate tem sido travado na área do raciocínio acerca das probabilidades ${ }^{3}$, tendo relação com o modo como a informação é representada na mente humana e, conseqüentemente, com a natureza dos processos disponíveis para se raciocinar e julgar com base em tais modos de representação. Em suma, Kahneman e Tversky propuseram que as pessoas tipicamente representam a informação em termos de exemplos individuais e, por conseguinte, julgam através de processos de raciocínio também baseados em exemplos individuais (abordagem dos vieses ou heurísticas). Contudo, Cosmides, Gigerenzer e colaboradores propuserem que os seres humanos costumam representar informação com base em freqüências populacionais, de modo que o raciocínio e julgamento desses últimos também são baseados em freqüências populacionais (abordagem freqüentista). Assim, enquanto para Kahneman e Tversky a probabilidade se refere, antes de tudo, a eventos únicos (expressando, assim, o nível de confiança subjetivo, i.e. de um determinado indivíduo acerca da verificação de um determinado evento), para Cosmides, Gigerenzer e seus associados, a probabilidade se refere exclusivamente a eventos de freqüencias populacionais.

A seguir, apresentar-se-ão estas duas abordagens, ressaltando-se, sobretudo, as implicações em termos de pesquisa. Antes, porém, de entrar no mérito deste debate, considera-se importante apresentar as questões e conceituar os termos tratados pelas duas abordagens supracitadas.

\section{O Raciocínio Probabilista}

Tradicionalmente, estuda-se o raciocínio probabilista relacionando-o com as teorias matemáticas da probabilidade. No início do desenvolvimento deste ramo da matemática, na segunda metade do século XVII e do século XVIII, as intuições de quem estava envolvido em tarefas que requeriam a avaliação de acontecimentos incertos (como no caso de apostas, jogos de azar entre outros) eram consideradas como fontes confiáveis dos cálculos formais de probabilidade. O matemático, físico e astrônomo francês Pierre-Simon de Laplace (1749-1827) contribuiu de forma considerável neste tipo de raciocínio, desenvolvendo a primeira teoria da probabilidade (Ex.: Teoria

\footnotetext{
${ }^{3} \mathrm{O}$ raciocínio de tipo probabilista diz respeito ao raciocínio indutivo, visto que este último tipo de raciocínio requer competências de tipo probabilista, como a capacidade de avaliar a variabilidade e a numerosidade de uma amostra a respeito da qual a inferência é realizada.
}

analitica da probabilidade, 1812). Laplace desenvolveu esta teoria através da inserção de um conjunto de técnicas de cálculo (funções geradoras) em um quadro de hipóteses capazes de fornecer dignidade autônoma à própria definição de probabilidade. Em sua obra Ensaio filosófico sobre a probabilidade (1814/1951), que se tornou em seguida em introdução das últimas edições da Teoria analítica, ele considerou criticamente os problemas que surgem a partir da aplicação do cálculo probabilista para o estudo dos fenômenos naturais. É de Laplace o ponto de vista "psicologizante" de que a teoria da probabilidade pode ser definida como simplesmente o senso comum reduzido a cálculo. Em um período caracterizado pelo ideal determinista, o recurso a critérios probabilísticos criou, de fato, inúmeros problemas que Laplace procurou superar recorrendo a uma inteligência "omnicompreensiva", a única capaz de ter certezas absolutas (nada seria incerto):

Given ... an intelligence which could comprehend all the forces by which nature is animated and the respective situation of the beings who compose it - an intelligence sufficiently vast to submit these data to analysis ... nothing would be uncertain and the future as the past, would be present to its eyes. (Laplace, 1814/1951, p. 4)

Ao invés de ser considerado como o resultado da formalização de julgamento intuitivo em condições de incertezas, o cálculo da probabilidade tem sido considerado por muitos psicólogos como uma forma ideal de avaliar as intuições. Conseqüentemente, os cálculos probabilísticos espontâneos têm sido considerados errados, na maioria dos casos, exatamente porque parecem divergir daqueles produzidos a partir da aplicação das regras probabilísticas normativas (Ex.: os utilizados para o cálculo da probabilidade).

Entretanto, nos últimos anos, tem ocorrido uma reação a esta tendência de avaliar os julgamentos probabilistas dos sujeitos “não expertos" tendo como parâmetros uma norma matemática, sobretudo em relação ao fato, geralmente pouco considerado pelos psicólogos, de que existem, em matemática, interpretações bastante diferentes sobre a probabilidade. Como resultado, mesmo sendo ainda precoce tirar conclusões definitivas desta reação, parece haver uma certa reabilitação das nossas capacidades de raciocinar em condições de incerteza.

Nesta perspectiva, o objetivo deste artigo é a) introduzir para reflexão este debate entre diferentes grupos de pesquisadores; b) apresentar recentes dados exploratórios coletados nesta área; e c) apontar possíveis linhas de investigações futuras que permitam uma melhor compreensão do problema. 


\section{O Teorema Bayesiano}

Um dos princípios estatísticos de base concerne à maneira pela qual é possível se estimar a probabilidade de um evento a partir de dois tipos de conhecimentos: não somente o que se sabe sobre tal evento antes que o mesmo se verifique, como também eventuais informações que podem ser obtidas em seguida. Isto é, tal princípio, formulado pelo reverendo matemático inglês Thomas Bayes (1702-1761), possibilita calcular a probabilidade de uma bipótese fundamentando-se na probabilidade a priori e em eventuais novas evidências relevantes.

Para tornar este princípio mais claro, considere-se o problema concreto abaixo - o problema da doença apresentado em duas partes:

Parte A: A prevalência da doença X é 1/1000. Um teste foi desenvolvido para detectar quando uma pessoa tem a doença X. Cada vez que o teste é realizado em uma pessoa que tem a doença, o resultado do teste é positivo.

Parte B: Mas, algumas vezes, o teste também apresenta um resultado positivo quando é realizado em uma pessoa que é completamente saudável. Especificamente, 5\% de todas as pessoas que são perfeitamente saudáveis apresentam um resultado positivo para a doença.

Questão: Qual é a chance de uma pessoa que teve o resultado positivo ter, de fato, a doença, assumindo que você não sabe de nada sobre os sintomas e sinais da pessoa?

Vamos considerar inicialmente a primeira parte do problema. A prevalência da doença X ser de 1/1000 indica a probabilidade a priori que define se uma determinada pessoa tem ou não a doença em questão (isto é, a incidência da doença na população; neste caso pode também ser definida como probabilidade de base ou primária ou proporção de base-em inglês base rate). Em outras palavras, se alguém perguntasse, anterior à realização do teste, uma estimativa da probabilidade de um indivíduo qualquer ter a doença $\mathrm{X}$, entre os que estão submetidos a um teste deste tipo, esta seria de 0,001 . Supondo-se que o teste realizado em um indivíduo tenha dado positivo (detecta a existência da doença $\mathrm{X}$ ), qual a probabilidade de que este indivíduo possua realmente a doença diagnosticada? Em termos mais formais, qual é a probabilidade a posteriori (i.e., o resultado da computação do teorema de Bayes) de ser a hipótese "O indivíduo tem a doença" verdadeira, visto que o teste deu positivo? Ou melhor, qual a probabilidade de um indivíduo, cujo teste tenha dado positivo, ser efetivamente doente?

Sem considerar a parte B do problema e pressupondo que o teste nunca falhe em seu diagnóstico, ou seja, que indique sempre a presença da doença quando o sujeito for efetivamente doente - definível como verdadeiro-positivo (em inglês a bit ou a true positive), e nunca indique a presença da doença quando o sujeito for, ao contrário, sadio (definível como falso-positivo (em inglês false positive ou false alarm), seria muito fácil estabelecer a probabilidade a posteriori de que a hipótese "o indivíduo tem a doença" fosse verdadeira, visto que o teste apresentou um resultado positivo. De fato, é possível concluir que um indivíduo diagnosticado como tendo a doença seja realmente doente.

Se considerarmos a parte B do problema, pressupondo, assim, de forma mais realista, que o teste às vezes apresente um resultado errado, a solução do problema torna-se mais difícil. Considere-se nosso exemplo, "algumas vezes o teste também apresenta um resultado positivo quando é realizado em uma pessoa que é completamente saudável”. Especificamente, $5 \%$ de todas as pessoas que são perfeitamente saudáveis apresentam um resultado positivo para a doença”; assim a taxa de casos falso-positivos é de $5 \%$, indicando que em 100 pessoas diagnosticadas como saudáveis, cinco têm a doença. A partir desta informação, é possível ser calculada a probabilidade de se obter um diagnóstico positivo: a) tanto na eventualidade em que o indivíduo é doente, b) como na eventualidade em que não é doente:

1) Uma pessoa é doente e o teste épreciso $100 \%$ das vezes para esta pessoa: Um indivíduo sobre 1000 é doente e se este indivíduo for submetido ao teste, a doença será certamente diagnosticada; em outras palavras, se uma pessoa é doente e o teste é capaz de detectar a doença em 100\% das vezes para este indivíduo, o cálculo da estimativa da probabilidade de que o teste seja positivo para um indivíduo doente é: 1 x 1,00=1,00

2) O restante 999 de nosso hipotético grupo de 1000 (i.e., com uma probabilidade $=0,999)$ não é doente e o teste é errado $5 \%$ das vezes para estas pessoas: Em caso de diagnóstico positivo na ausência da doença, temos a probabilidade de 0,04995, a qual é computada a partir da multiplicação da estimativa da probabilidade de base, da probabilidade de um sujeito ser sadio - 0,999 - pela probabilidade que o teste o indique como doente $-0,05$. Isto significa que iremos obter 49,95 indivíduos sadios - em um total de 1000 erroneamente diagnosticados como doentes. Isto é, com uma probabilidade da taxa de resultados de testes falsopositivos $=0,04995 ; 49,95$ indivíduos em uma população de 1000 seria erroneamente identificados como tendo a doença.

A partir da adição dos resultados destes dois cálculos, torna-se possível computar a probabilidade de se auferir um diagnóstico positivo, independentemente deste ser correto ou não: 0,001 +0,04995 =0,05095. Em termos de freqüências, é possível se afirmar que a doença é diagnosticada em aproximadamente 50,95 casos em um total de 1000, e que destes 50,95, enquanto 1 caso é diagnosticado 
corretamente, os outros 49,95 casos são diagnosticados de maneira errada.

A partir desta informação inicial, é possível computarmos a probabilidade a posteriori, a partir do teorema bayesiano da probabilidade, de ser um sujeito realmente doente, visto que tem sido diagnosticado como doente. Em outras palavras, é necessário avaliar nossa estimativa inicial da probabilidade de um indivíduo ter uma doença tendo como base o resultado do teste, considerando-se ao mesmo tempo a precisão deste teste em diagnosticar a doença.

Assim, o cálculo da probabilidade a posteriori (o indivíduo ter realmente a doença que lhe foi diagnosticada) é o resultado da razão entre duas estimativas de probabilidade:
Lógica e a Matemática - fornecem procedimentos computacionais normativos para se chegar à solução de vários tipos de problemas, o raciocínio comum tem pouco ou nenhum acesso a tais procedimentos e depende, ao invés disso, de uma série de heurísticas não-lógicas. O conceito de heurística foi introduzido por Simon $(1957,1982)$, em suas explorações sobre os limites do raciocínio dos seres humanos, para indicar algumas estratégias ao mesmo tempo econômicas e parcimoniosas, como também eficazes, mas não sistemáticas de resolução dos problemas. Tais heurísticas ou estratégias de pensamento são procedimentos não sistemáticos de tipo informal, aproximações práticas, ou então apenas estratégias gerais, mas não os algoritmos exatos fornecidos pelas teorias normativas. De fato, enquanto a ativação de uma heurística pode levar
Estimativa da probabilidade de diagnóstico positivo para um indivíduo doente (verdadeiro-positivo) Estim. da prob. de diag. positivo p/ um indivíduo doente (verdadeiro-positivo) + Estim. da prob. de diag. positivo $\mathrm{p} / \mathrm{um}$ indivíduo sadio (falso-positivo)
Estimativa da probabilidade de diagnóstico positivo para um indivíduo doente

= Estimativa da probabilidade total que seja diagnosticada uma doença (i.e., diag. posit. tanto p/ ind. doente como $\mathrm{p} /$ ind. sadio )

$\frac{(1 \times 1,00)}{(1 \times 1,00)+(999 \times 0,05)}=\frac{1}{1+49,95}=\frac{1}{50,95}=0,019627$

A partir deste cálculo, é possível respondermos à questão Qual é a chance de uma pessoa que teve o resultado positivo, de fato, ter a doença, assumindo que você não sabe de nada sobre os sintomas e sinais da pessoa?. Se a probabilidade a priori de nosso indivíduo ser doente era de 0,001 (1/1000) e, em seguida, após submetido a um teste, ter sido diagnosticado positivamente, a probabilidade a posteriori de ser realmente doente é de 0,19627. Em termos de freqüência, 1, em um total de 50,95 indivíduos que são testados como positivos, tem a doença; e expressado como uma freqüência relativa, pode-se dizer que entre todas as pessoas diagnosticadas como doentes, somente $1,9627 \%$ é, de fato, doente.

Esta descrição semi-intuitiva do teorema de Bayes pode ser representada mais formalmente. Entretanto, antes de apresentar esta representação formal do teorema, é importante introduzirmos a "abordagem dos vieses e das heurísticas". Esta abordagem argumenta que as pessoas ao invés de utilizarem os procedimentos computacionais do teorema bayesiano para solucionar problemas do tipo acima descrito, geralmente são incapazes de fazê-lo, utilizando, ao contrário, uma ampla variedade de atalhos não normativos e inadequados de tipo informal ao tomar de decisões.

\section{A Abordagem dos Viesses e das Heurísticas}

Daniel Kahneman e Amos Tversky, dois psicólogos de Israel que emigraram para a América do Norte, argumentaram que, enquanto disciplinas formais - como a a uma solução correta de um problema, sua aplicação não garante necessariamente que esta solução seja sempre alcançada; é possível que em outros casos esta mesma heurística seja capaz de produzir resultados diferentes daqueles que podem ser obtidos através da aplicação das regras probabilísticas normativas. Estes resultados diferentes consistem geralmente em erros sistemáticos.

Entre as heurísticas propostas por Kahneman e Tversky (1982; Tversky \& Kahneman, 1973, 1982) estão a representatividade, a prototipicalidade, a ancoragem e a disponibilidade, todas tendo em comum a sua dependência do uso de representações de casos individuais exemplares. Por exemplo, os julgamentos de probabilidade que um determinado evento ocorra podem depender da ativação de uma heurística como a da "disponibilidade", que nos leva a julgar como mais prováveis os eventos que são mais facilmente relembrados.

Vamos considerar o problema de Linda como exemplificação da heurística da representatividade (julgamentos influenciados pelo que é típico). Em um determinado estudo, Tversky e Kahneman (1982, 1983) apresentaram aos participantes uma rápida descrição de uma jovem chamada Linda:

Linda tem 31 anos, não é casada, é extrovertida e brilhante. É formada em filosofia e quando era estudante universitária era bastante engajada politicamente e participava de muitas manifestações antinucleares. 
Em seguida, apresentavam-se oito sentenças relativas às atividades de Linda e perguntava-se qual das sentenças descritivas de Linda mais provavelmente diziam a verdade a respeito da mesma. Mais especificamente, o sujeito devia ordenar as sentenças do nível mais provável para o nível menos provável. Abaixo são apresentadas três destas sentenças que o sujeito tinha que ordenar:

\section{Descrição A: "Linda é uma bancária"; \\ 2. Descrição B: "Linda é uma feminista"; \\ 3. Descrição A e B: "Linda é uma bancária e uma feminista".}

A maioria dos sujeitos escolhia a sentença 3 como mais provável do que as outras duas, embora a teoria da probabilidade canônica de eventos conjuntos afirme que a probabilidade de um conjunto isolado é sempre maior ou igual à probabilidade daquele conjunto em interseção com um outro ${ }^{4}$. Mais especificamente, aproximadamente 90\% dos sujeitos considerava a sentença 1 (Descrição A) Linda é uma bancária como menos provável do que a sentença 3 (Descrição A e B) Linda é uma bancária e uma feminista. Desta forma, os julgamentos dos sujeitos violam o principio estatístico e lógico de inclusão de classes, possibilitando a ocorrência ao erro ou falácia de conjunção (conjunction fallacy), isto é, uma avaliação da probabilidade de dois eventos conjuntos superiores à probabilidade de eventos isolados.

Segundo Tversky e Kahneman (1982), isso é uma evidência do uso de uma "heurística da representatividade", já que a idéia ou imagem geral de uma jovem que é uma feminista é mais representativa do que a idéia ou imagem de uma jovem que é uma bancária. Assim, ao compararmos a descrição Linda é uma bancária e uma feminista (A e B), menos provável, visto que menos específica, com a descrição Linda é uma bancária (A), a descrição "A e B" acaba sendo avaliada como mais provável do que a descrição "A" 5 , visto que o traço "ser feminista" representa adequadamente tudo o que se sabe sobre Linda, e, por isso, é mais representativo sobre ela. Assim, a heurística da representatividade de um evento pode levar as pessoas a uma avaliação errada da probabilidade de um evento ocorrer.

O problema de Linda é bem conhecido na literatura da área. Entretanto, existem outros dois problemas experimentais que introduziram, de maneira mais clara,

\footnotetext{
${ }^{4}$ Considerando A e B como eventos, a probabilidade $(\mathrm{P})$ de A ou B ocorrer é sempre maior ou igual à probabilidade de ocorrerem $\mathrm{A}$ e $\mathrm{B}$ ao mesmo tempo: $\mathrm{P}$ $(\mathrm{A}$ e $\mathrm{B}) \leq \mathrm{P}(\mathrm{A})$ ou $\mathrm{P}(\mathrm{B})$. Este princípio estatístico pode ser visto como uma forma específica do princípio lógico de inclusão de classes, de acordo com o qual um conjunto é sempre superior a/ou igual a soma de cada uma de suas partes. ${ }^{5}$ A possibilidade de que, neste caso em particular, a representatividade pode ser culturalmente específica é de pouca importância para fins da presente discussão.
}

o debate nesta área: o problema do táxi e o problema dos engenheiros/advogados. Estes dois problemas serão analisados mais detalhadamente a seguir.

Um dos primeiros problemas experimentais que levou ao debate é o problema do táxi, o qual se apresenta da seguinte forma:

Duas empresas de táxi operam numa dada cidade: a Táxi Azul e a Táxi Verde. $85 \%$ dos táxis na cidade são verdes, enquanto que os $15 \%$ restantes são azuis. Um táxi envolveuse em uma batida e fugiu durante a noite. Mais tarde, uma testemunha identificou o táxi como sendo azul. O tribunal examinou a capacidade da testemunha de distinguir entre os táxis verdes e azuis sob as condições noturnas de visibilidade. Foi constatado que a testemunha identificou cada cor corretamente em $80 \%$ das vezes e confundiu as duas cores em $20 \%$ das vezes. Quais são as chances de que o táxi criminoso tenha sido azul, como a testemunha relatou?

Kahneman e Tversky (1982) argumentaram que a solução normativamente correta de um problema deste tipo consiste em computar a probabilidade a posteriori que o táxi seja efetivamente azul, tendo como base a informação específica -i.e., a proporção de casos "verdadeiro-positivos" e "falsopositivos" de identificação de um táxi como azul, e da informação relativa à probabilidade de base. Assim, para estes autores, o procedimento computacional normativo para resolver este problema é fornecido pelo Teorema de Bayes, através do qual se pode calcular a probabilidade $a$ posteriori de que um evento A (azul = o táxi é azul) ocorra, dado que um evento B independente ("azul" = testemunha diz que o táxi é azul) também tenha ocorrido, bastando-se usar a fórmula abaixo:

$$
P(A \mid B)=\frac{P(A) P(B \mid A)}{P(A) P(B \mid A)+P(N \tilde{a} O A) P(B \mid N \tilde{a} O A)}
$$

$\mathrm{P}(\mathrm{A} \mid \mathrm{B}) \quad=$ Probabilidade $a$ posteriori de $\mathrm{A}$, dado que $\mathrm{B}$ tenha ocorrido

$\mathrm{P}(\mathrm{A}) \quad=\quad$ Probabilidade de $\mathrm{A}^{7}$

$\mathrm{P}(\mathrm{B} \mid \mathrm{A})=$ Probabilidade de $\mathrm{B}$, dado que $\mathrm{A}$ tenha ocorrido

$\mathrm{P}(\mathrm{NãoA})=$ Probabilidade de eventos $\mathrm{NãoA}^{8}$

$\mathrm{P}(\mathrm{B} \mid \mathrm{Não} \mathrm{A})=$ Probabilidade de $\mathrm{B}$, dado que Não $\mathrm{A}$ tenha ocorrido

${ }^{6}$ Eventos não-relacionados, que podem ou não ocorrer conjuntamente. Ser motorista de ônibus e gostar de algodão-doce, por exemplo, são eventos independentes. Já ser motorista de ônibus e ser cobrador não são independentes, pois há uma influência de um para com o outro (não se pode ser ambos ao mesmo tempo).

${ }^{7}$ No contexto de problemas de probabilidade condicional, também é conhecida como probabilidade de base ou primária, taxa básica, freqüência populacional ou base rate.

${ }^{8}$ Os eventos NãoA são todos os eventos que, juntos com A, representam a totalidade dos eventos dependentes possíveis. No exemplo do ônibus, se A for "ser motorista", então NãoA incluiria "ser cobrador" e "ser passageiro" ("algodão-doce" não entra em consideração por ser um evento independente). 
No caso do problema dos táxis, tem-se

$\begin{aligned} & P(\mathrm{~A} \mid \mathrm{B})= \begin{array}{l}\text { Probabilidade do táxi ser azul, dado que a } \\ \text { testemunha disse que ele é azul - probabilidade a } \\ \text { posteriori }\end{array} \\ & P(\mathrm{~A})=\begin{array}{l}\text { Probabilidade de um táxi ser azul - probabilidade } \\ \text { apriori }(0,15)\end{array} \\ & P(\mathrm{~B} \mid \mathrm{A})= \begin{array}{l}\text { Probabilidade da testemunha dizer que o táxi } \\ \text { é azul, dado que o táxi é de fato azul (likelihood) }\end{array} \\ &(0,80) \\ & P(\mathrm{Não})= \text { Probabilidade de um táxi ser verde }(0,85) \\ & P(\mathrm{~B} \mid \mathrm{NãoA})= \begin{array}{l}\text { Probabilidade da testemunha dizer que o táxi é } \\ \text { azul, dado que o táxi é de fato verde }(0,20)\end{array}\end{aligned}$

Assim, a fórmula calcula a razão da probabilidade dos táxis azuis que seriam identificados corretamente pela testemunha, dividido pela soma da probabilidade dos táxis azuis que seriam identificados corretamente, e da probabilidade dos táxis verdes identificados incorretamente como azuis, i.e., pela probabilidade total que um táxi seja identificado como azul, incluindo as identificações corretas e incorretas.

A seguir, são apresentadas as fórmulas com as notações - onde o azul (entre aspas) indica o evento "táxi identificado pela testemunha como azul”, enquanto azul e verde (sem aspas) indicam as cores reais dos táxis - e os valores fornecidos no enunciado do problema:

$$
P(a z u l \mid " a z u l ")=\frac{P(a z u l) P(" a z u l " \mid a z u l)}{P(a z u l) P(" a z u l " \mid a z u l)+P(\text { verde }) P(" a z u l " \mid \text { verde })}
$$$$
P(A \mid B)=\frac{(0,15 \times 0,80)}{[(0,15 \times 0,80)+(0,85 \times 0,20)]}=\frac{0,12}{0,12+0,17}=\frac{0,12}{0,29}=0,41
$$

Ou seja, com base nos dados disponíveis, ao contrário do que afirmado pela testemunha, existe uma chance de cerca de $41 \%$ dos táxis envolvidos no acidente serem azuis; fato que decorre do maior número de táxis verdes circulantes na cidade. Em termos freqüentistas, é possível afirmar que em um total de 29 táxis identificados como azuis (independentemente dos mesmos terem sido identificados corretamente ou não; 12 , de fato, azuis e 17, de fato, verdes), somente $41 \%$ serão efetivamente azuis.

Acontece que tal resposta quase nunca é encontrada nos estudos relatados na literatura. Ao invés disso, a resposta modal, normalmente apresentada por $40-50 \%$ dos sujeitos, é $80 \%$, que coincide com a atendibilidade da testemunha, i.e., com a capacidade de identificar corretamente como azul um táxi azul, ou melhor, a probabilidade da testemunha fazer uma identificação correta; não se considera, assim, o número de táxis em circulação (isto é, na avaliação da probabilidade a posteriori, não se considera a probabilidade de base ou primária - base rate - que corresponde ao percentual de táxis azuis e verdes em circulação) (Ex.: BarHillel, 1980; Birnbaum \& Mellors, 1983; Hammerton, 1973; Kahneman \& cols., 1982; Kahneman \& Tversky, 1973; Lyon $\&$ Slovic, 1976). Apenas uma pequena minoria de sujeitos costuma dar uma resposta que aparenta ser claramente influenciada pelas "probabilidades de base ou primária" (base-rates) dos táxis verdes e azuis - resultado este denominado como a "falácia da probabilidade de base" (base rate fallacy or base-rate neglect: falta de consideração da probabilidade de base). Aparentemente, esses achados não dependem do conteúdo específico do problema do táxi, tendo em vista que já foram replicados em problemas que se referem, por exemplo, ao diagnóstico dado por um mecânico de automóveis (Birnbaum \& Mellors, 1983) ou à identificação de indivíduos com uma dada doença a partir de um teste médico (Heller, Saltzstein \& Caspe, 1992).

A estratégia geral de Kahneman e Tversky tem sido a de apresentar problemas de raciocínio para os quais um juízo normativo pode ser definido e para os quais respostas que correspondam a tal juízo são raras. De acordo com a teoria, esses "erros sistemáticos" - que podem decorrer da ativação tanto de vieses como de uma ou mais heurísticas (ou atalhos) ${ }^{9}$ representam, portanto, a diferença entre o desempenho real e o normativamente correto. Esses erros serão, em seguida, explicados através de uma variedade de fórmulas heurísticas ou vieses de resposta, tais como representatividade (julgamentos influenciados pelo o que é típico), disponibilidade (julgamentos baseados no que é mais facilmente relembrado), prototipicalidade (julgamentos que se fundamentam em modelos prototípicos), ancoragem (julgamentos que se baseiam no que vem primeiro) e assim por diante. Uma maior compreensão desta variedade de fórmulas heurísticas possibilita determinar alguns dos erros sistemáticos envolvidos no raciocínio probabilístico.

As respostas apresentadas ao problema dos táxis são consistentes com o uso de heurísticas dessa natureza porque os sujeitos parecem se concentrar na informação individualizante, excluindo a informação que foi dada sobre as freqüências populacionais; uma tendência de resposta que seria de se esperar a partir de heurísticas individualizantes, tais como a representatividade ou a dependência de um protótipo. Em suma, nessa abordagem, a informação é

\footnotetext{
9 Para Kahneman e Tversky as heurísticas cognitivas podem estar tanto conexas com a especificidade da informação particular (Tversky \& Kahneman, 1980), como associadas à ausência de causalidade (Kahneman \& Tversky, 1980). Entre outros fatores explicativos do fenômeno observado podem ser citados a saliência (Nisbett \& Borgida, 1975) e a pertinência (BarHillel, 1983).
} 
representada em termos de exemplos individuais de conceitos significativos, o que resulta no uso de processos de pensamento que extraem inferências a partir desses exemplos.

Considera-se um outro problema, o dos engenheiros/ advogados elaborado por Kahneman e Tversky (1973). Neste problema, pede-se também ao sujeito para calcular a probabilidade a posteriori da ocorrência de um determinado evento que, apesar de ser bem menos complexo do que o problema do táxi ou da doença - anteriormente apresentados - parece, da mesma forma, produzir, em geral, respostas erradas peculiares.

Um grupo de psicólogos analisou a personalidade de 70 engenheiros e 30 advogados (condição 1; em segunda condição os engenheiros eram 30 e os advogados 70). A partir desta análise, os psicólogos elaboraram fichas descritivas de cada uma das 100 pessoas entrevistadas. A partir do total destas fichas, vão ser extraídas, ao acaso, cinco. Qual a probabilidade desta ficha descritiva pertencer a uma das duas categorias profissionais? (Esta probabilidade era avaliada em uma escala que variava de 0 a 100).

Um exemplo de ficha extraída é apresentado a seguir:

João tem 45 anos, é casado e tem quatro filhos. Tende a ser bastante conservador e é muito cuidadoso e ambicioso. Não possui qualquer interesse na área política e social e passa a maioria do seu tempo livre em atividades como fazer pequenos consertos, passear de barco à vela e se entreter em jogos matemáticos.

Para resolver este problema, é necessário considerar que a probabilidade de base que um indivíduo escolhido ao acaso, a partir da amostra, seja um engenheiro é muito diferente nas duas condições (70\% na condição 1, 30\% na condição 2). Entretanto, apesar da diferença na probabilidade de base, a maioria dos indivíduos não apresentava julgamentos de probabilidades diferentes nas duas condições. Geralmente, julgavam uma mesma probabilidade - que João fosse um engenheiro nas duas condições; mesmo quando os engenheiros eram menos (30\%), julgavam como mais provável que a ficha escolhida fosse a de um engenheiro. Se considerarmos este problema e o julgamento do ponto de vista estatístico, pode-se afirmar que os sujeitos entrevistados não consideravam a probabilidade de base e focalizavam a atenção para fornecer o julgamento probabilista nos dados relativos às fichas descritivas fornecidas pelo entrevistador. Por outro lado, quando se solicitava do sujeito julgar a probabilidade de um sujeito qualquer, escolhido ao acaso entre os dois grupos de profissionais sem a apresentação da ficha descritiva, ser um engenheiro, os sujeitos, ao responderem, consideravam a probabilidade de base, isto é, seus julgamentos se diferenciavam em função do número relativo de engenheiros iniciais (70 ou 30).

Para Tversky e Kahneman esta tendência em não considerar a probabilidade de base e consequentemente fornecer respostas erradas opostas àquelas que manifestam a tendência ao assim denominado "conservadorismo", que corresponde a uma sobreavaliação da probabilidade de base, é neste caso determinada pela heurística da "representatividade" (Tversky \& Kahneman, 1982). Através deste termo, como se pode inferir, define-se a relação de similaridade entre um indivíduo "X" e a classe " $Z$ " a qual pertence, ou entre uma amostra " $\mathrm{A}$ " e a população "P" da qual é retirada.

É neste ponto que, para estes pesquisadores, a heurística da representatividade impede um julgamento probabilista estatisticamente válido. Ao se julgar algo como representativo, isto é, que possui características típicas da classe à qual pertence, o julgamento sobre a probabilidade de que este algo pertença de fato à classe considerada será influenciado pelo grau de representatividade atribuída que, do ponto de vista estatístico, não é relevante, e ao mesmo tempo, deixarão de ser consideradas outras variáveis estatisticamente relevantes, como a probabilidade de base. No exemplo de João, ocorre exatamente o que foi descrito acima; visto que os traços que o definem parecem muito representativos do engenheiro típico, os entrevistados estarão mais propensos em considerá-lo um provável engenheiro independentemente de qualquer consideração da probabilidade de base.

Isto não significa que o grau de representatividade de algo em relação a uma classe não possa ser, em determinados casos, um bom índice para o julgamento da probabilidade de que o indivíduo pertença àquela própria classe. Geralmente, é mais provável que seja extraído, a partir de uma determinada população, um indivíduo com um alto nível de representatividade, em detrimento de um com um baixo nível de representatividade. Esta consideração não contrasta com o conceito de heurística, visto que uma heurística pode levar a uma rápida e correta solução de alguns tipos de problemas.

Uma outra heurística sugerida por Kahneman e Tversky é a da disponibilidade. Em um experimento realizado por estes dois pesquisadores (Tversky \& Kahneman, 1973), solicitava-se dos sujeitos avaliar a probabilidade ou freqüência de uma determinada letra do alfabeto $(K, L, N, R$ ou V) se encontrar no começo de uma palavra, comparando com a probabilidade, por exemplo, da mesma letra se encontrar na terceira posição. Apesar das cinco consoantes serem mais freqüentes na terceira posição, 2/3 dos participantes do 
estudo julgavam que a primeira posição era mais provável para a maioria das letras ${ }^{10}$. Isto se deve ao fato de que as pessoas fundamentam suas estimativas na disponibilidade relativa de palavras destes dois tipos, i.e., na facilidade de relembrar palavras que possuem a letra solicitada respectivamente na primeira e na terceira posição. Por exemplo, faz sentido pressupor ser mais fácil relembrar palavras que possuem a letra " $r$ " na primeira posição (Ex.: "rua"), comparativamente com palavras que possuem a mesma letra na terceira posição (Ex.: “cara"). Conseqüentemente, se basearmos nossas estimativas de probabilidade na disponibilidade relativa destas palavras, nossas estimativas serão erradas em todos aqueles casos ou eventos nos quais as freqüências reais são diferentes daquelas que podem ser calculadas, exatamente, de acordo com a disponibilidade.

Outras aplicações da heurística da disponibilidade podem ser observadas também em julgamentos que dizem respeito a eventos mais realistas do que o exemplo acima descrito sobre a freqüência relativa das palavras. Tversky e Kahneman (1973) têm encontrado que uma classe de elementos que podem ser relembrados com mais facilidade (Ex.: personagens famosas do mundo cinematográfico ou da música) era avaliada como mais numerosa do que uma classe do mesmo tamanho, mas contendo elementos menos fáceis de relembrar. Nesta mesma ótica, apontam os resultados de outros estudos, como o de Brown e Siegler $(1992,1993)$ na estimação de parâmetros demográficos como o tamanho da população de um país e o estudo de Lichenstein, Slovic, Fishhoff e Layman (1978) sobre a tendência das pessoas em exagerar a freqüência de algumas causas específicas de morte. Por exemplo, no estudo de Lichtenstein e colaboradores, foi encontrado que as causas de morte que são mais facilmente relembradas, visto serem mais divulgadas pelos meios de comunicação (televisão, jornais, revistas, etc.), devido a sua natureza mais espetacular e violenta, são superestimadas em relação à sua freqüência real (Ex.: mortes decorrentes de acidentes de avião, furacões). Ao contrário, outras causas de morte menos noticiadas, visto que são mais comuns e, por conseguinte, menos interessantes, são menos disponíveis e conseqüentemente subestimadas (Ex.: mortes decorrentes de cirrose hepática ou diabete). Todos estes estudos têm levado os pesquisadores a apontar que a heurística da disponibilidade é "one of he most widely shared assumptions in the decision making as well as in social judgment research" (Schwartz \& cols., 1991, p. 195).

\footnotetext{
${ }^{10}$ Para uma discussão mais aprofundada neste tópico ver também Lopes e Oden (1991) e Sedlmeier, Hertwig e Gigerenzer (1998).
}

De acordo com Kahneman e Tversky, as evidências acima apresentadas apontam para o fato de que, os casos nos quais é difícil relembrar todos os elementos de uma categoria em que precisa ser computada a freqüência relativa ou absoluta, as estimativas dos sujeitos se apoiam na facilidade ou melhor disponibilidade de acesso a exemplos significativos de tal categoria.

A abordagem teórica dos vieses e das heurísticas de Kahneman e Tversky e todo o programa de investigação realizado para corroborar esta posição teórica têm sido submetidos a um exame crítico e veementemente questionado duramente nos últimos anos por uma série de pesquisadores (Ex.: Cohen, 1981, 1986; Cosmides \& Tooby, 1996; Fiedler, 1988; Gigerenzer, 1991a, 1991b, 1996; Gigerenzer \& Hoffrage, 1995; Gigerenzer, Todd \& ABC Research Group,1999), argumentando que as leis da teoria da probabilidade podem ser compreendidas mais facilmente quando as estimativas de eventos incertos são solicitadas em termos de freqüências. Em outras palavras, seria mais fácil calcular probabilidades relativas a freqüências do que a eventos únicos ou singulares e, conseqüentemente, seria necessário fornecer informações freqüentistas para poder se obter julgamentos de probabilidade "bayesianamente" corretos.

\section{A Abordagem Freqüentista}

Gigerenzer e seus colegas (Ex.: Gigerenzer, 1991a, 1991b, 1993; Gigerenzer, Hell \& Blank, 1988; Gigerenzer \& Hug, 1992a; Gigerenzer \& cols., 1989) sugeriram que a aparente dificuldade de problemas, como o do táxi, e a conseqüente falta de consideração da probabilidade de base originamse de um erro conceitual fundamental cometido por Kahneman e Tversky quando eles propuseram o Teorema de Bayes como sendo o padrão normativo em relação ao qual o desempenho no problema do táxi deveria ser comparado. Para estes autores, uma visão da probabilidade como relacionada a eventos únicos não deve necessariamente ser considerada como a norma a respeito da qual se julgue o nível de desempenho de pessoas "não espertas" em estatística.

Assim, para estes autores, é possível formular uma interpretação alternativa à abordagem dos vieses: erros na solução do problema do táxi e em problemas similares ocorreram porque os problemas apresentavam informações e perguntavam questões sobre probabilidade de eventos únicos, ao invés de freqüências populacionais. Por exemplo, se a questão do problema do táxi fosse, não sobre a probabilidade de um único táxi ser azul, mas sobre a freqüência pela qual táxis identificados como azuis terem sido identificados corretamente, seria mais provável as 
pessoas fornecerem respostas em consonância com o teorema Bayesiano.

Após uma pesquisa na literatura canônica sobre a teoria das probabilidades, Gigerenzer e seus colegas argumentaram que, nas intuições das pessoas comuns, as probabilidades se referem a freqüências populacionais ao invés de eventos isolados ${ }^{11}$. É mais natural estimar as probabilidades de eventos relativos a freqüências populacionais do que estimar a probabilidade de eventos únicos. Em outras palavras, eles afirmam que a mente é estatisticamente freqüentista. Dado isso, se for pedido às pessoas que julguem a freqüência de uma população ao invés da probabilidade de um caso único, elas são mais propensas a prestarem atenção à informação sobre a freqüência ou probabilidade de base (base rate) e chegar a uma solução que corresponda ao output do Teorema de Bayes, desde que os pressupostos usuais acerca da aleatoriedade de uma amostra sejam atendidos.

Cosmides e Tooby (Ex.: Cosmides \& Tooby, 1996) juntaram-se ao debate tomando a postura freqüentista, acrescentando, a partir da psicologia evolucionista, um argumento a seu favor com base em suposições acerca das origens bioevolucionárias da mente moderna. Uma das principais preocupações da psicologia evolucionista concerne em estabelecer quais podem ter sido as perguntas cognitivas através das quais o meio teria desafiado o ser humano no decorrer da evolução de sua espécie. Para estes autores, a partir de uma análise deste tipo, a psicologia evolucionista deveria ser capaz de estabelecer quais seriam as competências cognitivas inatas dos atuais representantes da espécie humana.

Nesta mesma perspectiva, para os freqüentistas, as pessoas normalmente usariam processos dependentes de conteúdo e raramente raciocinariam de forma dirigida por princípios normativos da lógica - ver, por exemplo, a Teoria do Contrato Social (Ex.: Cosmides, 1989; ver também Gigerenzer \& Hug, 1992a, 1992b). ${ }^{12}$

\footnotetext{
${ }^{11}$ Uma probabilidade pode ser vista como se referindo a um fenômeno que ocorrerá uma única vez - um evento isolado - ou como se referindo a uma fração dos eventos dentro de um universo de ocorrências - uma frequiência relativa.

${ }^{12}$ A Teoria do Contrato Social propõe alguns módulos de raciocínio derivados da bio-evolução que as pessoas usam para identificar os violadores de contratos sociais condicionais universalmente quantificados regulando custos e benefícios; nesta perspectiva Cosmides escreveu que as pessoas não pensam "de acordo com os cânones de lógica" (p. 191). A Teoria dos Esquemas de Raciocínios Pragmáticos (Ex.: Cheng \& Holyoak, 1985), por sua vez, propôs alguns módulos de raciocínio indutivamente adquiridos que as pessoas presumidamente usam para identificar os violadores de obrigações condicionais e permissões; neste sentido Cheng, Holyoak, Nisbett e Oliver (1986) debateram que as pessoas raramente, caso isso ocorra, pensam de acordo com regras lógicas. Embora as produções dos propostos processos de conteúdo-específico correspondam ao que se seguiria de uma lógica normativa padrão, a partir das perspectivas destas propostas tais
}

Através desta conjugação de argumentos freqüentistas e suposições bioevolucionárias, Cosmides tem argumentado que os ancestrais humanos, que viviam em sociedades de caçadores/coletores, teriam representado informações num formato freqüentista ao invés de um formato probabilista. Mais especificamente, a partir do pressuposto de que uma capacidade simples e elementar de raciocínio estatístico tenha evoluído nos tempos através de um processo de seleção natural, é necessário supor que o seu primeiro campo de aplicação em uma sociedade não letrada estivesse relacionado com dados em formato de freqüências, i.e. representações de casos discretos como "4 sobre 32" e não uma probabilidade como $p=0,125$, ou também percentuais como $12,5 \%$.

Neste mesmo sentido, Gigerenzer (1993) aponta que teriam sido necessários vários milênios de história do pensamento matemático antes que a moderna teoria da probabilidade a respeito de eventos únicos fosse desenvolvida. Conseqüentemente, para os partidários da abordagem freqüentista, a maioria dos erros de raciocínio e julgamento probabilista apontados na literatura da área não poderiam ser mais considerados como erros. Isto é, se se supõe que a capacidade de raciocínio das pessoas comuns ocorre em condições de incerteza avaliando as probabilidades de ocorrência de freqüências de eventos e não de ocorrência de eventos únicos, então será preciso julgar as capacidades estatísticas comuns através de critérios freqüentistas.

\section{As Dúbias Evidências a Favor da Abordagem Freqüentista}

Tanto Gigerenzer e Hoffrage (1995) quanto Cosmides e Tooby (1996) apresentaram dados para dar suporte às suas previsões de que as pessoas farão juízo normativamente adequados acerca de probabilidades condicionais desde que os problemas apresentem informação do modo freqüentista (ao invés do probabilista) e que requeiram

respostas não serão encontradas em situações onde os processos de conteúdo-específico não se aplicam, e quando o raciocínio realmente corresponder às normas de um sistema de lógica, isto representa mera coincidência. Teóricos do conteúdo-específico não estão sozinhos quando argumentam que processos de raciocínio-dedutivo não correspondem a uma lógica normativa. Johnson-Laird e seus colaboradores argumentaram que o raciocínio usa modelos mentais ao invés de quaisquer regras de lógica (Ex.: Johnson-Laird \& Byrne, 1991, 1992, 1993; Johnson-Laird, Byrne \& Schaeken, 1992). Evans e seus associados argumentam que as pessoas aplicam uma variedade de vieses de raciocínio e heurísticas ao invés de regras lógicas (Ex.: Evans, 1989; Evans, Newstead, \& Byrne, 1993). No que se refere a argumentar sobre probabilidades, Kahneman e Tversky (Ex.: 1973, 1982, 1996) semelhantemente propuseram que as pessoas normalmente são incapazes de fazer julgamentos que corresponda ao que seria computado usando-se a teoria de probabilidade normativa e depender, por outro lado, de uma variedade de vieses de raciocínio e heurísticas. 
julgamento acerca de freqüências populacionais (ao invés de probabilidades de eventos únicos). Tais dados, porém, não fornecem suporte inequívoco à interpretação freqüentista.

Uma discussão extensa de todos os problemas e comparações não é possível aqui, tendo em vista que Gigerenzer e Hoffrage apresentaram 30 problemas no primeiro experimento enquanto Cosmides e Tooby apresentaram oito. Contudo, os dois problemas discutidos abaixo são representativos dos tipos de dificuldade que se encontram ao longo desses grandes conjuntos de problemas:

Problema Freqüentista: É dito aos participantes que 10 de cada 1000 mulheres com 40 anos de idade que participaram de um exame de rotina tinham câncer de mama. Oito de cada 10 mulheres com câncer de mama terá uma mamografia positiva 95 de cada 990 mulheres sem câncer de mama também terão uma mamografia positiva. Aqui está uma nova amostra representativa de mulheres com 40 anos que têm uma mamografia positiva num exame de rotina. Quantas dessas mulheres você acha que, realmente, têm câncer de mama? de

Problema Probabilista: É dito aos participantes que a probabilidade de câncer de mama na população é de $1 \%$. Se uma mulher tem câncer de mama, há $80 \%$ de probabilidade de que ela tenha uma mamografia positiva, mas se ela não tiver câncer, há uma probabilidade de 9,6\% de que ela terá uma mamografia positiva. Qual a probabilidade de que uma mulher que tem uma mamografia positiva, de fato, tenha câncer? $\%$

Gigerenzer e Hoffrage relataram que a solução correta (8 de 103) é obtida significativamente mais vezes na versão freqüentista do que na versão probabilista $(0,078$, isto é, $7,8 \%$ ). Será que se pode concluir, como eles fizeram, que a razão para esse resultado é o fato de que a primeira versão apresentou informação freqüentista e uma pergunta sobre freqüência populacional, enquanto que a segunda apresentou informação probabilista e uma pergunta sobre a probabilidade de um evento único?

A conclusão de Gigerenzer e Hoffrage sobre a causa dos resultados acima é prejudicada pelo fato de que os dois problemas diferem em várias formas que nada tem a ver com a variável independente, ou seja, os formatos probabilistas e freqüentistas. Primeiramente, o problema freqüentista apresenta toda a informação numérica em números inteiros, mas a tarefa probabilista apresenta parte da informação através de frações decimais. Em segundo lugar, enquanto o problema freqüentista apresenta o campo de resposta num formato que sugere o uso de uma razão (i.e., de __ ) o problema probabilista não o faz (i.e., $\%$. De fato, devido à diferença no formato de resposta, o problema probabilista exige uma etapa de cálculo a mais do que o freqüentista. Além disso, há ainda a questão de que os valores necessários para se computar a resposta bayesiana ( 8 e 95 , permitindo que se compute 8 de [8+95]) são fornecidos diretamente na versão freqüentista, enquanto que o valor de 0,008 precisa ser computado a partir dos valores de $1 \%$ e $80 \%$ que são dados para a população e as taxas verdadeiro-positivas no formato probabilista (de modo que se possa computar $[0,01 \times 0,80] /$ $[[0,01 \mathrm{x} 0,80]+0,096])$. Assim, a versão probabilista difere e parece ser mais difícil do que a versão freqüentista, ocorrendo isso em várias dimensões que nada tem a ver com as diferenças de interesse teórico (i.e., se as informações e perguntas concernem a probabilidades ou freqüências). Claramente, a demanda computacional da versão probabilista é maior do que da versão freqüentista.

A partir do conjunto destas observações, é possível se extrair duas conclusões. Primeiro, Gigerenzer e Hoffrage (1995) e Cosmides e Tooby (1996) têm relatado várias versões de problemas nos quais as pessoas costumeiramente apresentam respostas bayesianas, assim demonstrando que Kahneman e Tversky estavam errados em sua afirmativa de que as pessoas carecem das habilidades necessárias para a produção de respostas a problemas que requerem o julgamento de probabilidades condicionais. Segundo, que, por causa dos múltiplos fatores de confusão presentes nos problemas empregados nas várias investigações realizadas pelos freqüentistas, não se pode concluir que a razão para o sucesso nas versões freqüentistas dos problemas tenha qualquer coisa a ver com a apresentação freqüentista ou probabilista das questões. De fato, O'Brien, Roazzi e Dias (no prelo) relataram que a proporção de soluções corretas diminui para quase zero quando o formato de resposta do tipo “___sobre___ é removido dos problemas apresentados na condição freqüentista, e, ao contrário, a proporção de respostas corretas aumenta para níveis comparáveis com problemas freqüentistas quando este formato de resposta é adicionado aos problemas apresentados na condição probabilista. Além do mais, O'Brien e colaboradores relatam que problemas freqüentistas são solucionados numa proporção bem menor quando números inteiros são substituídos por frações decimais, e, ao mesmo tempo, a proporção de soluções corretas aumenta quando as frações decimais dos problemas probabilistas são substituídos com números inteiros. Estes resultados sugerem fortemente, e com bastante segurança, que as evidências relatadas por Gigerenzer e Hoffrage (1995) em favor da hipótese freqüentista devem ter, certamente, resultado de procedimentos metodológicos inadequados e bastante questionáveis. 
Em acréscimo às comparações empíricas mal planejadas que são reveladas pelo exemplo do problema da mamografia, existem outras razões para se duvidar de que os registros de pesquisa existentes permitam que se faça um juízo claro acerca dos formatos representacionais e das habilidades de raciocínio no contexto das probabilidades. Os tipos de problemas de laboratório que normalmente são apresentados nas investigações não abordam as intuições básicas acerca da probabilidade. Ao contrário, eles são bastante complexos e lançam mão de um amplo número de processos. Além disso, tais processos são mal definidos, no sentido de que a pesquisa não foi planejada para abordar as contribuições diferenciais de natureza aritméticas, estratégicas, conteúdodependentes e assim por diante. Em suma, não se sabe o fator responsável por tornar os problemas mais difíceis, nem o que faz com que os indivíduos adotem certas linhas de raciocínio, tampouco o que determina a tendência das pessoas de se concentrarem mais em determinadas informações do problema do que em outras.

\section{O Argumento Bioevolucionário para a Abordagem Freqüentista}

Como mencionado, uma importante justificativa teórica para a proposta freqüentista baseia-se em pressupostos bioevolucionários. Cosmides e Tooby (1996) partem do princípio de que as únicas estatísticas disponíveis para os ancestrais humanos do período Pleistocênico, caçadores/ coletores, eram fundamentadas na observação pessoal ou advinham de um pequeno número de outros indivíduos que faziam parte de um grupo. Tendo em vista que não se pode observar a probabilidade de um evento - simplesmente, ou ele ocorre ou não - conclui-se que é impossível para a natureza criar um órgão sensorial para detectar probabilidades de eventos únicos: "Nenhum organismo pode desenvolver mecanismos cognitivos projetados para raciocinar sobre, ou receber como input, informações num formato que não existisse regularmente" (Cosmides \& Tooby, p. 15).

Diferentemente das probabilidades, as quais se afirmavam ser completamente separadas da observação, as freqüências eram tidas por Cosmides e Tooby como sendo diretamente abertas à observação - as freqüências estão "disponíveis no ambiente" (p. 15). Os ancestrais humanos que eram caçadores/coletores podiam observar, por exemplo, que 5 das últimas 20 expedições de caças realizadas em determinado local tiveram sucesso. Como dizem Cosmides e Tooby,

nossos ancestrais hominídeos estavam imersos num rico fluxo de freqüências observáveis, as quais poderiam ser utilizadas para melhorar a tomada de decisões, sendo fornecidos os procedimentos que pudessem tirar vantagem delas. Então, sendo verdade que temos adaptações para o raciocínio indutivo, elas devem tomar informação sobre freqüências como input (pp. 15-16) $)^{13}$

O que se deveria fazer com essa afirmativa de que "as freqüências existem ao nível das observações, enquanto que as probabilidades são tão completamente divorciadas da experiência que é conceitualmente impossível para a natureza produzir quaisquer formatos representacionais ou procedimentos de raciocínio que sejam baseadas nelas"? Essa noção foi apresentada por Cosmides e Tooby sem qualquer justificativa adicional, como se simplesmente fosse óbvia sob o ponto de vista intuitivo. Contudo, a afirmativa parece ser duvidosa quando se faz uma inspeção mais aprofundada. Embora a idéia de que eventos únicos podem estar abertos à observação direta pareça filosoficamente plausível, ainda é preciso alguma forma de registro desses eventos da experiência na memória, assim como uma forma de contar esses eventos armazenados, para se saber o quão freqüentemente eventos de um determinado tipo têm ocorrido. Não se deve confundir a observação de eventos com a observação das suas freqüências. Mesmo excluindo o fato de que cada evento é único, e que, conseqüentemente, é preciso uma forma de categorizar os eventos de modo a se saber o que incluir quando se calcula uma freqüência, é bastante óbvio que é necessário se computar algo para se ter uma contagem de freqüência.

Desta maneira, da mesma forma que não se deveria afirmar que probabilidades ocorrem no nível da observação, também não se deveria afirmar que freqüências ocorrem assim. Tanto as freqüências quanto as probabilidades requerem memória, computação e formas de categorização, sendo que nenhuma delas se dá por observação.

Cosmides e'Tooby não apenas argumentaram que somente as freqüências são abertas à observação direta e, conseqüentemente, à representação direta, mas eles também afirmaram que as pessoas não convertem as freqüências em probabilidades após as primeiras “have been picked up” (p. 16); isso se dá por que as freqüências incluem a probabilidade de base das observações e "quando ' $n$ ' desaparece, o índice de confiabilidade da informação desaparece também” (p. 16). Além do mais, eles argumentaram que, na medida em que novas observações são realizadas, a base de dados pode ser atualizada. Por exemplo, após uma caçada fracassada, os ancestrais humanos caçadores/coletores podiam atualizar a informação de que 5 de 20 caçadas tiveram sucesso

\footnotetext{
${ }^{13} \mathrm{O}$ raciocínio indutivo é o raciocínio através do qual são tiradas conclusões gerais a partir de premissas especificas ou através do qual se passa de proposições especificas para outras proposições especificas através de proposições gerais. Inúmeras são as atividades de pensamento que fazem uso deste tipo de raciocínio, como por exemplo, a formação de conceitos, as predições, as generalizações tiradas a partir de exemplos entre outros.
} 
para a de que 5 de 21 tiveram sucesso. Isso parece presumir algumas habilidades aritméticas da parte dos ancestrais do período Pleistocênico, as quais podem estar tipicamente ausentes numa sociedade de caçadores/coletores, onde tanto os números cardinais quanto ordinais são passíveis de grande limitação, freqüentemente se restringindo a contar "1", “2", “3” e "mais".

Embora a afirmativa freqüentista seja a respeito das bases bioevolucionárias das intuições e, assim sendo, acerca das necessidades cognitivas presumidas para os ancestrais humanos do período Pleistocênico, os problemas de laboratório que, de fato, têm sido apresentados dependem de uma série de habilidades especializadas típicas de culturas "desenvolvidas". Até mesmo os tipos de habilidades que seriam exigidas para a existência das capacidades descritas por Cosmides e Tooby (1996) como ocorrendo entre os ancestrais humanos caçadores/coletores também parecem ser altamente desenvolvidas (Ex.: a habilidade aritmética de estabelecer razões com denominadores grandes). Finalmente, apesar do fato de que todas as proposições teóricas oriundas desses debates têm feito afirmativas acerca de algo universal ao ser humano, raramente quaisquer dessas investigações incluem participantes outros que não sujeitos universitários. Particularmente interessante é o fato de que nenhum dos dados coletados tem a ver com indivíduos de sociedades de caçadores/coletores.

\section{Então, o que investigadores cognitivos deveriam fazer para que estes tópicos façam sentido?}

Há três pontos que a pesquisa relativa a estas questões precisa urgentemente esclarecer. Primeiro, precisa ser estabelecido se (ou não) o efeito encontrado pelos freqüentistas é real. Embora vários autores tenham questionado recentemente as evidências e conclusões propostas pelos freqüentistas (Lewis \& Keren, 1999; Mellers \& McGraw, 1999; Vranas, 2000), Gigerenzer e Hoffrage (1999) têm afirmado "There seems to be a consensus about the effects' existence. The debate has now shifted to the questions of why the effect occurs and what its boundary conditions are" (p. 425). Esta afirmação de que o efeito freqüentista já pode ser considerado como algo estabelecido e não mais questionável, parece-nos claramente prematura. Os problemas de natureza metodológica nas comparações entre problemas freqüentistas e problemas probabilistas acima descritos não esclarecem o fato de que um formato freqüentista seja superior a um formato probabilista.

Em uma recente investigação de O'Brien e colaboradores (no prelo) foram encontradas evidências mostrando claramente que os resultados apresentados por Gigerenzer e Hoffrage (1995) e por Cosmides e Tooby (1996) são resultados de falhas metodológicas sérias como, por exemplo, a presença versus ausência de um "formato de resposta" e o uso de números inteiros nas versões dos problemas na condição freqüentista versus o uso de frações decimais nas versões dos problemas na condição probabilista. Realmente, O'Brien e colaboradores argumentam que os resultados relatados por parte dos autores acima não podem ser explicados assumindo que as pessoas estavam chegando a respostas de tipo Bayesiana através de uma linha de raciocínio Bayesiana como Gigerenzer, Cosmides e pesquisadores associados fizeram - mas podem também ser explicados muito bem de forma similar assumindo que os problemas apresentados na condição freqüentista possibilitavam que os participantes pudessem fazer uso de estratégias de adivinhação que os levavam a produzir respostas corretas; estratégias estas que pelo formato dos problemas apresentados, infelizmente, não eram disponibilizadas na condição probabilista. Esta possível estratégia, que tem sido denominada por O’Brien e colaboradores de “dumbluck theory" ("teoria da sorte de tolo"), torna real a possibilidade de que toda uma linha de investigação desenvolvida nas últimas duas décadas nesta área tenha pressuposto falsamente que respostas corretas derivem de raciocínios apropriados, enquanto respostas incorretas não. O'Brien e colaboradores levantam a possibilidade de que talvez tanto as respostas corretas como as incorretas não tenham se originado, de forma alguma, de linhas de raciocínio Bayesianas.

Segundo, se alguém for considerar seriamente os argumentos bioevolucionários apresentados por Cosmides e Tooby (1996) e Gigerenzer (1998), seria de suma importância descobrir quais habilidades de raciocínio e representacionais realmente existem em sociedades de caçadores/coletores. Se Kahneman e Tversky estiverem corretos, então os membros de uma sociedade de caçadores e coletores pré-históricos, por exemplo os Amondava, deveriam revelar a fortiori uma falta de habilidades de raciocínios normativamente apropriados, embora se tivesse uma expectativa de poder encontrar algumas habilidades que representassem casos individuais e, posteriormente, fazer generalização sobre elas. Por outro lado, se os freqüentistas estiverem corretos, conseqüentemente, deveríamos encontrar pelo menos algumas representações primitivas e habilidades de raciocínio por freqüência, mas não por probabilidade.

Entretanto, como foi apontado anteriormente, o argumento Bioevolucionário tem sido altamente especulativo e seus autores não se referiram a qualquer dado de populações caçadoras/coletoras existentes. Além do mais, os argumentos especulativos nos parecem ser suspeitos, como argumentado acima, fundamentado-se no pressuposto 
de que enquanto as probabilidades derivariam de computações, as freqüências não; porém, claramente, tanto as probabilidades como as freqüências derivam basicamente de computações. Todavia, a ausência de qualquer referência para dados levantados em sociedades de caçadores/coletores nos deixa um tanto perturbados, especialmente porque tal coleta de dados é possível. Muito recentemente foi descoberto que sociedades de caçadores/coletores existem na Amazônia brasileira, tornando, assim, possível a verificação dos tipos de habilidades que estas populações possuem para se representarem tanto as freqüências como as probabilidades. Claro que, a partir do momento em que os investigadores voltarem suas atenções para populações deste tipo, é improvável que eles continuem apresentando os tipos de problemas geralmente usados em situações experimentais controladas de tipo laboratorial que foram descritos anteriormente, i.e., com exigências aritméticas e computacionais complexas. A habilidade para computar divisões longas, por exemplo, requer indubitavelmente um treinamento educacional formal que naturalmente não se espera ser encontrado em populações caçadoras/coletoras indígenas. Isto não significa, evidentemente, que tais pessoas são incapazes de fazer julgamentos sobre probabilidades ou sobre freqüências. Porém, significa que é necessário que sejam apresentadas situações computacionais menos exigentes para descobrir quais são estas habilidades.

Em terceiro lugar, investigações de laboratório do tipo que são realizadas em contextos universitários deveriam começar a incluir problemas que não apresentam o tipo de exigências aritméticas e matemáticas/computacionais que têm se tornado dominantes na literatura previamente publicada. Isto é verdade não somente para o tipo de coleta de dados com populações caçadoras-coletoras que nós sugerimos há pouco serem necessárias, mas também para coleta de dados com estudantes universitários do tipo normalmente utilizado na literatura.

O interesse teórico de todos os investigadores que estão envolvidos nesta área de pesquisa é descobrir o formato representacional básico e os processos de raciocínio que fazem parte do repertório cognitivo. Ninguém tem proposto que o interesse nestes estudos está em descobrir se o sistema educacional teve êxito ou não no ensino do teorema de Bayes ou de outros conceitos e procedimentos computacionais matemáticos sofisticados. A pesquisa de Kahneman e Tversky, de um lado, e de Gigerenzer, Cosmides, e colaboradores, de outro, diz respeito aos formatos representacionais básicos e aos processos de raciocínio ao invés de formas de representar e processar informação que dependam de instrução formal. Entretanto, participantes em nossos estudos freqüentemente respondem a estes tipos de problemas com comentários do tipo: "Ainda não fiz o curso no qual nos ensinam a resolver este tipo de problemas", "Eu realmente não me lembro do que é preciso fazer para resolver este tipo de problema", ou "Nunca fui bom em matemática". Por outro lado, e em número bem menor, existem aqueles participantes que afirmam com orgulho que conseguiram resolver o problema porque relembraram os procedimentos de solução destes tipos de problemas em algum curso.

Enfim, é importante ressaltar o fato que os tipos de problemas usualmente utilizados nesta área de pesquisa nunca serão capazes de descobrir processos básicos. Se alguém os quiser descobrir, sugerimos que se comece a incluir a apresentação de problemas de raciocínio que não demandem dos participantes conhecimentos de procedimentos matemáticos sofisticados cuja presença somente escamoteia a existência de processos cognitivos básicos. Se estes cuidados na elaboração dos problemas não forem tomados, avanços substanciais na compreensão destes processos dificilmente ocorrerão.

\section{Referências}

Bar-Hillel, M. (1980). The base-rate fallacy in probability judgments. Acta Psychologia, 44, 211-233.

Birnbaum, M. H. \& Mellors, B. A. (1983). Bayesian inference: Combining base rates with opinions of sources who vary in credibility. Journal of Personality and Social Psychology, 45, 792-804.

Brown, N. R. \& Siegler, R. S. (1992). The role of availability in the estimation of national populations. Memory \& Cognition, 20, 406-412.

Brown, N. R. \& Siegler, R. S. (1993). Metrics and mappings: A framework for understanding real-world quantitative estimation. Psychological Review, 100, 511-534.

Cheng, P. \& Holyoak, K. J. (1985). Pragmatic reasoning schemas. Cognitive Psychology, 17, 391-416.

Cheng, P. \& Holyoak, K. J. (1989). On the natural selection of reasoning theories. Cognition, 33, 285-313.

Cheng, P., Holyoak, K. J., Nisbett, R. E. \& Oliver, L. M. (1986). Pragmatic vs. syntactic approaches to training deductive reasoning. Cognitive Psychology, 18, 293-328.

Cohen, L. J. (1981). Can human irrationality be experimentally demonstrated? Behavioral and Brain Sciences, 4, 317-331.

Cohen, L. J. (1986). The dialogue of reason: An analysis of analytical philosophy. Oxford: Clarendon Press.

Cosmides, L. (1989). The logic of social exchange: Has natural selection shaped how humans reason? Studies with wason selection task. Cognition, 31, 187276.

Cosmides, L. \& Tooby, J. (1989). Evolutionary psychology and the generation of culture, part II. Case study: A computational theory of social exchange. Ethology and Sociobiology, 10, 51-97.

Cosmides, L. \& Tooby, J. (1996). Are humans good intuitive statisticians after all? Rethinking some conclusions from the literature on judgment under uncertainty. Cognition, 58, 1-73.

Evans, J. St. B. T. (1982). The psychology of deductive reasoning. London: Routledge \& Kegan Paul.

Evans, J. St. B. T. (1989). Bias in human reasoning: The fragmented state of the art. Theory and Psychology, 1, 83-105.

Evans, J. St. B. T., Newstead, S. E. \& Byrne, R. M. J. (1993). Human reasoning: The psychology of deduction. Hove, UK: Lawrence Erlbaum. 
Fiedler, K. (1988). The dependence of the conjunction fallacy on subtle linguistic factors Psychological Research, 50, 123-129.

Gigerenzer, G. (1991a). From tools to theories: A heuristic of discovery in cognitive psychology. Psychological Review, 98, 254-267.

Gigerenzer, G. (1991b). How to make cognitive illusions disappear: Beyond heuristics and biases. Em W. Stroebe \& M. Hewstone (Orgs.), European review of Social Psychology (Vol. II, pp. 83-115). New York: Wiley.

Gigerenzer, G. (1993). The bounded rationality of probabilistic mental models. Em K. Manktelow \& D. Over (Orgs.), Rationality (pp. 145-169). London: Routledge.

Gigerenzer, G. (1996). On narrow norms and vague heuristics: A reply to Kahneman and Tversky (1996). Psychological Review, 103, 592-596.

Gigerenzer, G. (1998). Ecological intelligence: An adapation to frequencies. Em D. D. Cummins \& C. Allen (Orgs.), The evolution of mind (pp.53-77). New York: Oxford University Press.

Gigerenzer, G. \& Hoffrage, U. (1995). How to improve Bayesian reasoning with instruction: Frequency formats. Psychological Review, 102, 684-704.

Gigerenzer, G. \& Hug, K. (1992a). Domain-specific reasoning: Social contracts, cheating, and perspective change. Cognition, 43, 127-171.

Gigerenzer, G. \& Hug, K. (1992b). Reasoning about social contracts: Cheating and perspective change. Cognition, 43, 127-171.

Gigerenzer, G. \& Hoffrage, U. (1999). Overcoming difficulties in Bayesian reasoning: A reply to Lewis and Keren (1999) and Mellers and McGraw (1999). Psychological Revien, 106, 425-430.

Gigerenzer, G., Hell, W. \& Blank, H. (1988). Presentation and content: The use of base rates as a continuous variable. Journal of Experimental Psychology: Human Perception and Performance, 14, 513-525.

Gigerenzer, G., Swijtink, Z., Porter, T., Daston, L., Beatty, J. \& Krüger, L. (1989). The empire of chance: How probability changed science and everyday life. Cambridge, Cambridge University Press.

Gigerenzer, G., Todd, P. M. \& ABC Research Group (1999). Simple heuristics that make us smart. Oxford: Oxford University Press.

Hammerton, M. (1973). A case of radical probability estimation. Journal of Experimental Psychology, 101, 242-254.

Heller, R. F., Saltzstein, H. D. \& Caspe, W. B. (1992). Heuristics in medical and nonmedical decision-making. Quarterly Journal of Experimental Psychology, 44a, 211-235.

Johnson-Laird, P. N. \& Byrne, R. M .J. (1991). Deduction. Hillsdale, NJ: Lawrence Erlbaum.

Johnson-Laird, P. N. \& Byrne, R. M. J. (1992). Modal reasoning, models, and Manktelow \& Over. Cognition, 43, 173-182.

Johnson-Laird, P. N. \& Byrne R. M. J. (1993). Mental models or formal rules? Behavioural and Brain Sciences, 16, 368-376.

Johnson-Laird, P. N., Byrne, R. M. J. \& Schaeken, W. (1992). Propositional reasoning by models. Psychological Review, 99, 418-439.

Kahneman, D. \& Tversky, A. (1972). Subjective probability: A judgment of representativeness. Cognitive Psychology, 3, 430-454.

Kahneman, D. \& Tversky, A. (1973). On the psychology of prediction. Psychological Review, 80, 237-251.

Kahneman, D. \& Tversky, A. (1982). The simulation heuristic. Em D. Kahneman, P. Slovic \& A. Tversky (Orgs.), Judgement under uncertainty: Heuristics and biases (pp. 69-91). Cambridge: Cambridge University Press.

Kahneman, D. \& Tversky, A. (1996) On the reality of cognitive illusions: A replay to Gigerenzer's critique. Psychological Revien, 103, 582-591.

Kahneman, D., Slovic, P. \& Tversky, A. (1982). Judgments under uncertainty: Heuristics and biases. New York: Cambridge University Press.
Laplace, P. S. (1951). A philosopbical essay on probabilities. New York: Dover. (Original publicado em 1814)

Lewis, C. \& Keren, G. (1999). On the difficulties underlying Bayesian reasoning: A comment on Gigerenzer and Hoffrage. Psychological Review, 106, 411-416.

Lichenstein, S., Slovic, P., Fishhoff, B. \& Layman, M. (1978). Judged frequency of lethal events. Journal of Experimental Psychology: Human Learning and Memory, 4,551-578.

Lopes, L. L. \& Oden, G. D. (1991). The rationality of intelligence. Em E. Eels \& T. Maruszewki (Orgs.), Poznan studies in the philosophy of the sciences and the humanities. (Vol. 21, pp. 225-249). Amsterdam: Rodopi.

Lyon, D. \& Slovic, P. (1976). Dominance of accuracy information and neglect of base rates in probability estimation. Acta Psychologia, 40, 287-298.

Mellers, B. A. \& McGraw, A. P. (1999). How to improve Bayesian reasoning: Comment on Gigerenzer and Hoffrage (1995). Psychological Review, 106, 417-424.

Nisbett, R. E. \& Borgida, E. (1975). Attribution and the psychology of prediction. Journal of Personality and Social Psychology, 32, 932-943.

O’Brien, D., Roazzi, A. \& Dias, M. G. B. B. (no prelo). Raciocínio sobre probabilidade condicionais: As evidências a favor da hipótese freqüentista se fundamentam em comparações errôneas. Estudos de Psicologia.

Schwartz, N., Bless, H., Strack, F., Klumpp, G., Rittenauer-Schatka, H. \& Simons, A. (1991). Ease of retrieval as information: Another look at the availability heuristic. Journal of Personality and Social Psychology, 61, 195-202.

Sedlmeier, P., Hertwig, R. \& Gigerenzer, G. (1998). Are judgments of the positional frequencies of letters systematically biased due to availability? Journal of Experimental Psychology: Learning, Memory, and Cognition, 24, 754-770.

Simon, H. A. (1957). Models of man: Social and rational. New York: Wiley.

Simon, H. A. (1982). Models of bounded rationality. Cambridge, MA.: MIT Press.

Tooby, J. \& Cosmides, L. (1989). Evolutionary Psychology and the generation of culture, Part I. Theoretical considerations. Ethology and Sociobiology, 10, 29-49.

Tversky, A. \& Kahneman, D. (1973). Availability: A Heuristic for Judging Frequency and Probability Cognitive Psychology, 5, 207-232.

Tversky, A. \& Kahneman, D. (1980). Causal schemas in judgments under uncertainty. Em M. Fishbein (Org.), Progress in social psychology (pp.108132). Hillsdale N.J.: Erlbaum.

Tversky, A. \& Kahneman, D. (1982). Judgments of and by representativeness. Em D. Kahneman, P. Slovic \& A. Tversky (Orgs.), Judgement under uncertainty: Heuristics and Biases (pp.127-1156). Cambridge: Cambridge University Press.

Tversky, A. \& Kahneman, D. (1983). Extensional versus intuitive reasoning: The conjunction fallacy in probability judgment. Psychological Review, 90, 293-315.

Vranas, P. B. M. (2000). Gigerenzer's normative critique of Kahneman and Tversky. Cognition, 76, 179-193.

Sobre os autores

Antonio Roazzi é Doutor pela Universidade de Oxford, Inglaterra. É Professor do Programa de Pósgraduação em Psicologia da Universidade Federal de Pernambuco.

David O'Brien é Professor do Baruch College and Graduate Center of the City University of New York. Maria da Graça Bompastor Borges Dias é Doutora pela Universidade de Oxford, Inglaterra. É Professora do Programa de Pós-graduação em Psicologia da Universidade Federal de Pernambuco. 